\title{
Wechsel im Schriftleiter-Team der Kinderrheumatologie
}

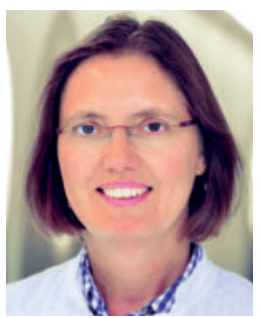

Prof. Dr. Almut Meyer-Bahlburg

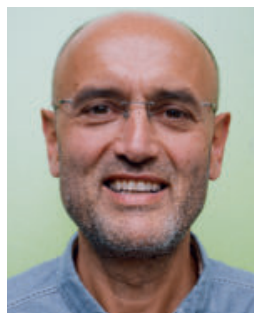

Dr. Toni Hospach

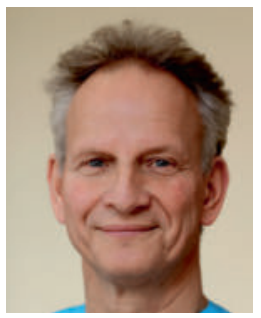

Frank Weller-Heinemann

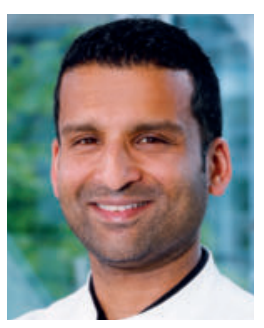

Dr. Prasad Thomas Oommen
Mit dem neuen Jahr findet ein Wechsel im Schriftleiter-Team der Kinderrheumatologie statt. Frau Prof. Kirsten Minden, die bisher gemeinsam mit Frau Prof. Almut Meyer-Bahlburg diese Funktion innehatte, wird sich nach 8 Jahren zurückziehen und anderen Aufgaben zuwenden. Es folgen ihr nach: Dr. Toni Hospach aus dem Olgahospital Stuttgart, Frank Weller-Heinemann aus der Professor-Hess-Kinderklinik Bremen sowie Dr. Prasad Thomas Oommen aus dem Universitätsklinikum Düsseldorf.

Unser besonderer Dank gilt an dieser Stelle allerdings zunächst Frau Prof. Kirsten Minden. Sie hat die Rubrik Kinderrheumatologie in der „arthritis + rheuma“ mit viel Engagement als 1. Schriftleiterin seit 2011 aufgebaut und geleitet und so für die „Gesellschaft für Kinder- und Jugendrheumatologie“ (GKJR) ein wichtiges Medium nach innen und außen etabliert.

Das neue Schriftleiter-Team möchte die bewährte Mischung aus lehrreichen Fällen, Übersichtsartikeln und Verbandsnachrichten aus der Fachgesellschaft im Grundsatz gerne beibehalten. Als fachliche Neuerung wollen wir darüber hinaus eine neue Rubrik „Kinderrheumatologie kompakt“ einführen (erstmals in der vorliegenden Ausgabe). Hier sollen kurze Zusammenfassungen aktueller Artikel zu Themen der Kinder- und Jugendrheumatologie aus wichtigen Journals zusammengefasst sowie herausragende Publikationen unserer Mitglieder vorgestellt werden. Hier ist die Schriftleitung für Hinweise zu besonderen Publikationen dankbar.

Gleich im ersten Heft hat sich Herr Dr. Andreas Urban aus Amberg mit einer Gruppe bayerischer Kinder- und Jugendrheumatologen das wichtige Thema „Biosimilars in der Kinder- und Jugendrheumatologie“ vorgenommen. Entstanden ist ein wichtiges Statement, in dem ein differenzierter und realistischer Umgang mit diesen Substanzen gefordert wird. Darüber hinaus präsentieren Christine Zachries et al. von der Prof.-Hess-Kinderklinik Bremen den Fall einer septischen Gonarthritis und eines ausgedehnten Wadenabszesses durch Streptococcus intermedius bei einem 4-jährigen Jungen.

So freuen wir uns, mit einem neuen Team dieses wichtige Organ unserer Fachgesellschaft ab 2020 zu begleiten. Die Rubrik lebt und entwickelt sich vor allem durch die Fälle und Artikel, die von Ihnen eingereicht werden.

Ihre

Prof. Dr. Almut Meyer-Bahlburg, Greifswald

Dr. Toni Hospach, Stuttgart

Frank Weller-Heinemann, Bremen

Dr. Prasad Thomas Oommen, Düsseldorf 